Supporting Information for

\title{
A Synthetic Approach to Nomofungin/Communesin B
}

\author{
Seth L. Crawley and Raymond L. Funk* \\ Department of Chemistry, Pennsylvania State University, \\ University Park, PA 16802
}<smiles>CCOC(=O)C(O)c1ccccc1O</smiles><smiles>CCOC(=O)C1OC(C)(C)Oc2ccccc21</smiles>

Ethyl 2,2-dimethyl-4H-benzo[1,3] dioxine-4-carboxylate. To a solution of ethyl hydroxy(2-hydroxyphenyl)acetate ${ }^{1}(4)(2.00 \mathrm{~g}, 10.2 \mathrm{mmol})$ in acetone $(4.4 \mathrm{~mL})$ was added 2,2-dimethoxypropane $(6.3 \mathrm{~mL}, 50.9 \mathrm{mmol})$ and $( \pm)$-camphorsulfonic acid (592 $\mathrm{mg}, 2.54 \mathrm{mmol}$ ). The solution was stirred at room temperature $12 \mathrm{~h}$, quenched with saturated aqueous sodium bicarbonate and extracted with ethyl acetate. The combined extracts were dried $\left(\mathrm{Na}_{2} \mathrm{SO}_{4}\right)$ and concentrated to provide a yellow oil $(2.00 \mathrm{~g}, 83 \%) ;{ }^{1} \mathrm{H}$ $\operatorname{NMR}\left(200 \mathrm{MHz}, \mathrm{CDCl}_{3}\right) \delta 1.31(\mathrm{t}, J=7.2 \mathrm{~Hz}, 3 \mathrm{H}), 1.52(\mathrm{~s}, 3 \mathrm{H}), 1.63(\mathrm{~s}, 3 \mathrm{H}), 4.27$ (q, $J=7.2 \mathrm{~Hz}, 2 \mathrm{H}), 5.45(\mathrm{~s}, 1 \mathrm{H}), 6.81(\mathrm{dd}, J=0.9,8.4 \mathrm{~Hz}, 1 \mathrm{H}), 6.91(\mathrm{dt}, J=0.9,8.4 \mathrm{~Hz}, 1$ $\mathrm{H}), 7.16(\mathrm{dt}, J=0.6,7.5 \mathrm{~Hz}, 1 \mathrm{H}), 7.29(\mathrm{~d}, J=7.5 \mathrm{~Hz}, 1 \mathrm{H}) ;{ }^{13} \mathrm{C} \mathrm{NMR}\left(50 \mathrm{MHz}, \mathrm{CDCl}_{3}\right)$ $\delta 14.0,23.0,26.9,61.6,70.3,99.4,116.7,117.4,120.6,125.1,129.1,150.9,169.4$; IR (neat) $2993,2941,1759,1732,1612,1587 \mathrm{~cm}^{-1}$; HRMS $\left(\mathrm{M}+\mathrm{H}^{+}\right)$calcd for $\mathrm{C}_{13} \mathrm{H}_{17} \mathrm{O}_{4}$ 237.1121, found 237.1134.<smiles>CCOC(=O)C1OC(C)(C)Oc2ccccc21</smiles>

2,2-Dimethyl-4H-benzo[1,3] dioxine-4-carboxylic acid. To a solution of ethyl 2,2dimethyl-4H-benzo[1,3]dioxine-4-carboxylate $(1.71 \mathrm{~g}, 7.23 \mathrm{mmol})$ in methanol $(18.1$ $\mathrm{mL})$ at $0{ }^{\circ} \mathrm{C}$ was added $1 \mathrm{M} \mathrm{KOH}(18.1 \mathrm{~mL}, 18.1 \mathrm{mmol})$ dropwise. The solution was stirred at $0{ }^{\circ} \mathrm{C}$ for $3 \mathrm{~h}$, quenched with potassium phosphate buffer $(\mathrm{pH}=5)$ and extracted with $\mathrm{Et}_{2} \mathrm{O}$. The combined organic extracts were dried $\left(\mathrm{Na}_{2} \mathrm{SO}_{4}\right)$ and concentrated to afford a white solid: $\mathrm{mp} 76-78^{\circ} \mathrm{C}(1.37 \mathrm{~g}, 91 \%) ;{ }^{1} \mathrm{H}$ NMR $\left(200 \mathrm{MHz}, \mathrm{CDCl}_{3}\right) \delta 1.53(\mathrm{~s}$, $3 \mathrm{H}), 1.65(\mathrm{~s}, 3 \mathrm{H}), 5.49(\mathrm{~s}, 1 \mathrm{H}), 6.83(\mathrm{~d}, J=8.0 \mathrm{~Hz}, 1 \mathrm{H}), 6.95(\mathrm{dt}, J=0.8,7.6 \mathrm{~Hz}, 1 \mathrm{H})$, 
$7.24(\mathrm{dt}, J=0.8,8.0 \mathrm{~Hz}, 1 \mathrm{H}), 7.47(\mathrm{~d}, J=7.6 \mathrm{~Hz}, 1 \mathrm{H}) ;{ }^{13} \mathrm{C} \mathrm{NMR}\left(50 \mathrm{MHz}, \mathrm{CDCl}_{3}\right) \delta$ 22.6, 27.1, 69.7, 99.7, 115.5, 117.5, 121.1, 125.3, 129.5, 150.7, 173.7; IR (neat) 3044, 2996, 1731, 1610, 1587, $1488 \mathrm{~cm}^{-1}$; HRMS $\left(\mathrm{M}+\mathrm{Na}^{+}\right)$calcd for $\mathrm{C}_{11} \mathrm{H}_{13} \mathrm{O}_{4} 231.0627$, found 231.0629 .
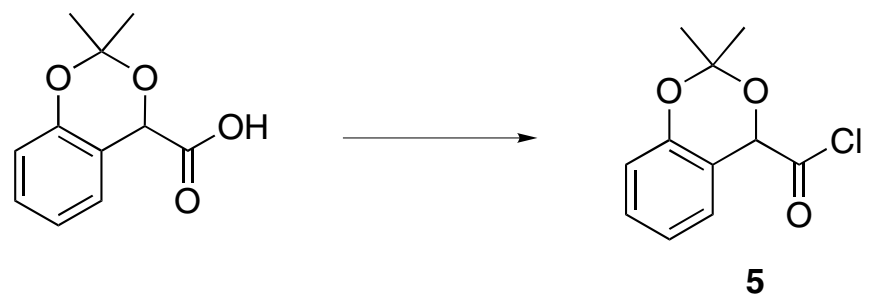

2,2-Dimethyl-4H-benzo[1,3]dioxine-4-carbonyl chloride (5). To a solution of 2,2dimethyl-4H-benzo[1,3]dioxine-4-carboxylic acid $(93.0 \mathrm{mg}, 0.447 \mathrm{mmol})$ in $\mathrm{CH}_{2} \mathrm{Cl}_{2}(1.5$ $\mathrm{mL})$ at $0{ }^{\circ} \mathrm{C}$ was added oxalyl chloride $(77.9 \mu \mathrm{L}, 0.893 \mathrm{mmol})$ and DMF $(3.5 \mu \mathrm{L}, 0.045$ mmol). The solution was stirred 5 min and concentrated in vacuo to provide a brown solid, which was used immediately without purification; ${ }^{1} \mathrm{H}$ NMR $\left(200 \mathrm{MHz}, \mathrm{CDCl}_{3}\right)$ $\delta 1.54(\mathrm{~s}, 3 \mathrm{H}), 1.65$ (s, $3 \mathrm{H}), 5.54(\mathrm{~s}, 1 \mathrm{H}), 6.93(\mathrm{~d}, J=8.2 \mathrm{~Hz}, 1 \mathrm{H}), 6.99$ (dt, $J=1.0,8.2$ $\mathrm{Hz}, 1 \mathrm{H}), 7.25(\mathrm{~d}, J=8.5 \mathrm{~Hz}, 1 \mathrm{H}), 7.29(\mathrm{t}, J=8.5 \mathrm{~Hz}, 1 \mathrm{H}) ;{ }^{13} \mathrm{C} \mathrm{NMR}\left(50 \mathrm{MHz}, \mathrm{CDCl}_{3}\right)$ $\delta$ 23.8, 26.4, 76.9, 100.1, 114.4, 117.9, 121.3, 125.6, 130.2, 151.0, 174.1.

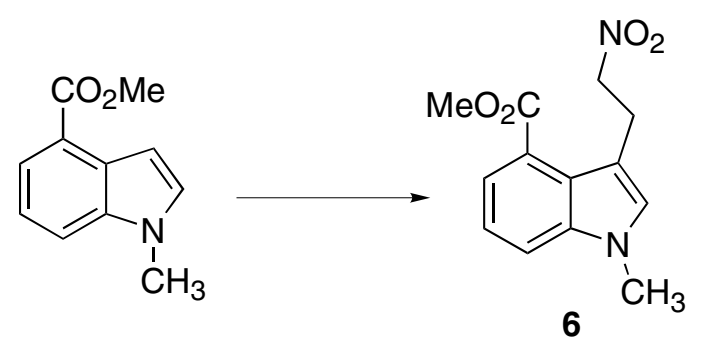

Methyl 1-methyl-3-(2-nitroethyl)-4-indolecarboxylate (6). The title compound was synthesized following the procedure of Clark for the preparation of methyl 3-(2nitroethyl)-4-indolecarboxylate ${ }^{2}$ To a solution of methyl 1-methyl-4-indolecarboxylate ${ }^{3}$ $(1.02 \mathrm{~g}, 5.37 \mathrm{mmol})$ in xylenes $(3.8 \mathrm{~mL})$ was added 2-nitroethyl acetate ${ }^{4}(764 \mathrm{mg}, 5.74$ $\mathrm{mmol})$, and 4-tert-butylcatechol $(17.8 \mathrm{mg}, 0.107 \mathrm{mmol})$. The solution was heated at 150 ${ }^{\circ} \mathrm{C}$ for $3 \mathrm{~h}$. The mixture was concentrated and purified by silica-gel chromatography $(1$ : 3 ethyl acetate : hexanes) to afford a white solid : $\mathrm{mp} 86-88{ }^{\circ} \mathrm{C}(923 \mathrm{mg}, 70 \%) ;{ }^{1} \mathrm{H}$ NMR (200 MHz, CDCl $\left.{ }_{3}\right) \delta 3.64(\mathrm{t}, J=6.6 \mathrm{~Hz}, 2 \mathrm{H}), 3.75(\mathrm{~s}, 3 \mathrm{H}), 3.95(\mathrm{~s}, 3 \mathrm{H}), 4.69$ (t, $J$ $=6.6 \mathrm{~Hz}, 2 \mathrm{H}), 7.04(\mathrm{~s}, 1 \mathrm{H}), 7.24(\mathrm{~d}, J=7.9 \mathrm{~Hz}, 1 \mathrm{H}), 7.5(\mathrm{dd}, J=1.0,7.9 \mathrm{~Hz}, 1 \mathrm{H}), 7.82$ $(\mathrm{dd}, J=1.0,7.9 \mathrm{~Hz}, 1 \mathrm{H}) ;{ }^{13} \mathrm{C} \mathrm{NMR}\left(50 \mathrm{MHz}, \mathrm{CDCl}_{3}\right) 25.8,32.6,51.9,77.4,109.2$, 114.0, 120.6, 122.9, 123.3, 131.3, 138.3, 168.2 ; IR (neat) 2949, 1714, 1609, 1555, 1454 $\mathrm{cm}^{-1}$; HRMS $\left(\mathrm{M}+\mathrm{H}^{+}\right)$calcd for $\mathrm{C}_{13} \mathrm{H}_{15} \mathrm{~N}_{2} \mathrm{O}_{4}$ 263.1026, found 263.1038. 

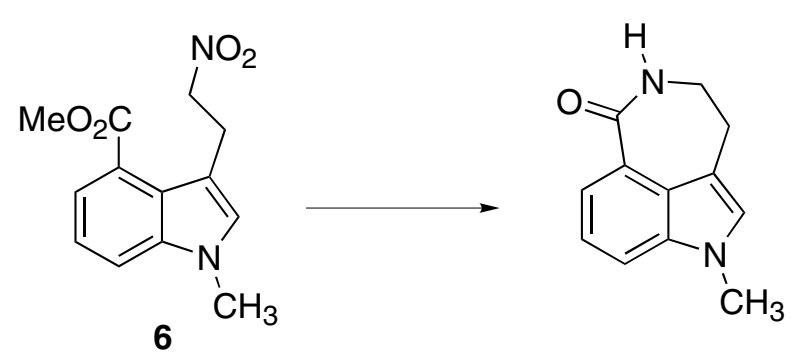

1-Methyl-1,3,4,5-tetrahydro-azepino[5,4,3-cd] indol-6-one. The title compound was synthesized following the procedure of Strekowski for the preparation of the analogous des $N$-methyl indole. ${ }^{5}$ To a solution of ester $6(923 \mathrm{mg}, 3.72 \mathrm{mmol})$ in ethanol $(12.4 \mathrm{~mL})$ was added platinum oxide $(21 \mathrm{mg}, 0.093 \mathrm{mmol})$. The mixture was stirred at $70{ }^{\circ} \mathrm{C}$ under a hydrogen atmosphere for $4 \mathrm{~d}$. The solution was filtered through celite and concentrated. Further purification by silica-gel chromatography $\left(33: 1 \mathrm{CH}_{2} \mathrm{Cl}_{2}: \mathrm{MeOH}\right)$ provided a light yellow solid: $\mathrm{mp} 179-180{ }^{\circ} \mathrm{C}(452 \mathrm{mg}, 61 \%) ;{ }^{1} \mathrm{H}$ NMR $(200 \mathrm{MHz}$, $\left.\mathrm{CDCl}_{3}\right) \delta 3.03(\mathrm{~m}, 2 \mathrm{H}), 3.59(\mathrm{dd}, J=5.6,9.7 \mathrm{~Hz}, 2 \mathrm{H}), 3.77(\mathrm{~s}, 3 \mathrm{H}), 6.92(\mathrm{~s}, 1 \mathrm{H}), 6.99$ (br s, $1 \mathrm{H}), 7.32(\mathrm{~d}, J=7.5 \mathrm{~Hz}, 1 \mathrm{H}), 7.43(\mathrm{~d}, J=7.5 \mathrm{~Hz}, 1 \mathrm{H}), 7.97(\mathrm{~d}, J=7.5 \mathrm{~Hz}, 1$ $\mathrm{H}) ;{ }^{13} \mathrm{C}$ NMR $\left(50 \mathrm{MHz}, \mathrm{CDCl}_{3}\right) \delta 28.2,32.6,43.2,112.9,114.2,121.3,122.9,124.3$, 125.5, 126.5, 137.1, 171.7; IR (neat) 3278, 2923, 1643, 1604, $1463 \mathrm{~cm}^{-1}$; HRMS $\left(\mathrm{M}+\mathrm{H}^{+}\right)$calcd for $\mathrm{C}_{12} \mathrm{H}_{13} \mathrm{~N}_{2} \mathrm{O} 201.1022$, found 201.1037.
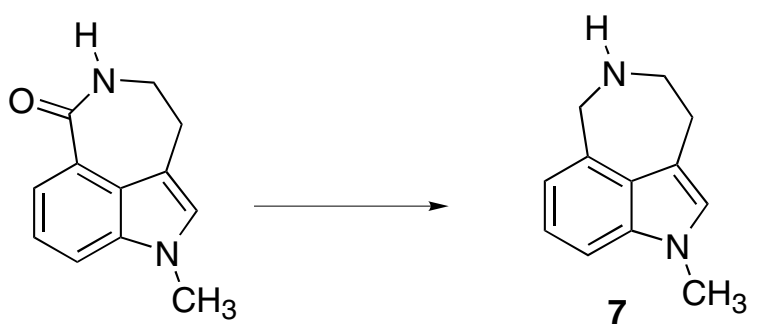

1-Methyl-3,4,5,6-tetrahydro- $1 H$-azepino[5,4,3-cd $]$ indole (7). The title compound was prepared following the procedure of Evans for the preparation of the analogous des $\mathrm{N}$ methyl indole. ${ }^{6}$ To a refluxing solution of lithium aluminum hydride $(135 \mathrm{mg}, 3.57$ mmol) in THF $(5.9 \mathrm{~mL})$ was added dropwise 1-methyl-1,3,4,5-tetrahydro-azepino[5,4,3$c d$ ] indol-6-one $(135 \mathrm{mg}, 0.674 \mathrm{mmol})$ in THF $(1.1 \mathrm{~mL})$. The solution was stirred at reflux for $3 \mathrm{~h}$. The mixture was quenched with water $(135 \mu \mathrm{L}), 10 \% \mathrm{NaOH}(202 \mu \mathrm{L})$, and water $(405 \mu \mathrm{L})$. The slurry was filtered and concentrated to give a light yellow solid: mp 64-66 ${ }^{\circ} \mathrm{C}(120 \mathrm{mg}, 95 \%) ;{ }^{1} \mathrm{H}$ NMR $\left(200 \mathrm{MHz}, \mathrm{CDCl}_{3}\right) \delta 2.15$ (br s, $\left.1 \mathrm{H}\right), 3.06(\mathrm{~m}, 2$ $\mathrm{H}), 3.21(\mathrm{~m}, 2 \mathrm{H}), 3.75(\mathrm{~s}, 3 \mathrm{H}), 4.35(\mathrm{~s}, 2 \mathrm{H}), 6.88(\mathrm{~m}, 2 \mathrm{H}), 7.17(\mathrm{~m}, 2 \mathrm{H}) ;{ }^{13} \mathrm{C}$ NMR (50 $\left.\mathrm{MHz}, \mathrm{CDCl}_{3}\right) \delta 31.1,32.2,50.5,56.1,106.6,113.7,115.4,120.9,125.5,125.7$, 136.7, 137.3; IR (neat) 2913, 1460, $1418 \mathrm{~cm}^{-1}$; HRMS $\left(\mathrm{M}+\mathrm{H}^{+}\right)$calcd for $\mathrm{C}_{12} \mathrm{H}_{15} \mathrm{~N}_{2}$ 187.1229, found 187.1177 . 

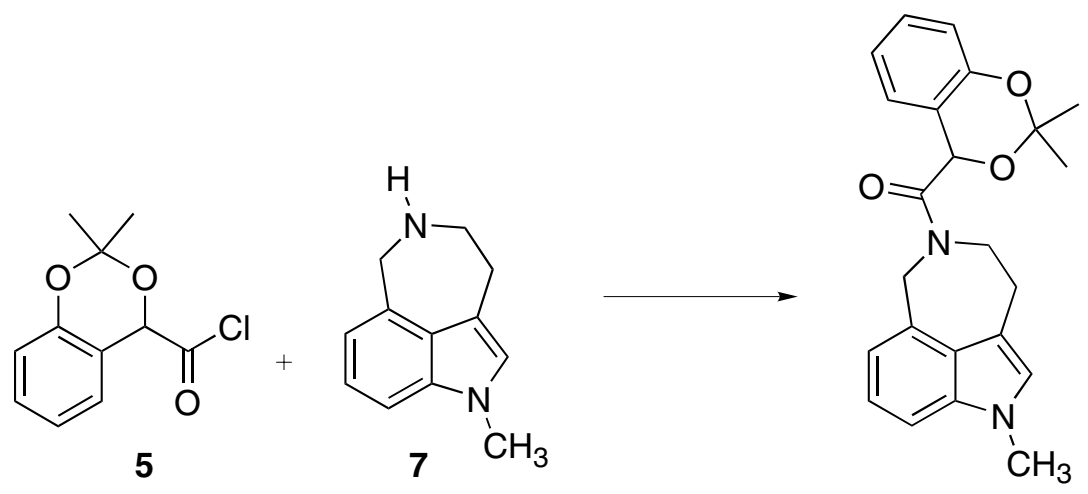

Acylation of amine 7 with acid chloride 5. To a solution of the amine 7 (220 mg, $1.18 \mathrm{mmol})$ and triethylamine $(430 \mu \mathrm{L}, 3.07 \mathrm{mmol})$ in $\mathrm{CH}_{2} \mathrm{Cl}_{2}(2.9 \mathrm{~mL})$ at $0{ }^{\circ} \mathrm{C}$ was added dropwise the acid chloride $5(401 \mathrm{mg}, 1.77 \mathrm{mmol})$ in $\mathrm{CH}_{2} \mathrm{Cl}_{2}(500 \mu \mathrm{L})$. The solution was stirred $5 \mathrm{~min}$, quenched with saturated aqueous sodium bicarbonate, and extracted with $\mathrm{CH}_{2} \mathrm{Cl}_{2}$. The combined organic extracts were dried $\left(\mathrm{Na}_{2} \mathrm{SO}_{4}\right)$, filtered, and concentrated. Purification by silica-gel chromatography $\left(33: 1 \mathrm{CH}_{2} \mathrm{Cl}_{2}: \mathrm{MeOH}\right)$ afforded a colorless oil as a mixture of rotamers $(300 \mathrm{mg}, 70 \%) ;{ }^{1} \mathrm{H}$ NMR $\left(200 \mathrm{MHz}, \mathrm{CDCl}_{3}\right) \delta 1.41$ (s, major, 3 $\mathrm{H}), 1.47$ (s, major, $3 \mathrm{H}), 1.54$ (s, minor, $3 \mathrm{H}$ ), 1.69 (s, minor, $3 \mathrm{H}), 3.21$ (t, $J=5.0 \mathrm{~Hz}, 2$ $\mathrm{H}), 3.74(\mathrm{~s}, 3 \mathrm{H}), 3.85(\mathrm{~m}, 1 \mathrm{H}), 4.03(\mathrm{~m}, 1 \mathrm{H}), 4.84(\mathrm{~m}$, minor, $2 \mathrm{H}), 4.82(\mathrm{~d}$, major, $J=$ $17.2 \mathrm{~Hz}, 1 \mathrm{H}), 4.93$ (d, major, $J=17.2 \mathrm{~Hz}, 1 \mathrm{H}), 5.67$ (s, major, $1 \mathrm{H}), 5.78$ (s, minor, 1 $\mathrm{H}), 6.67(\mathrm{t}, J=7.9 \mathrm{~Hz}, 2 \mathrm{H}), 6.86(\mathrm{~m}, 2 \mathrm{H}), 6.96(\mathrm{~d}, J=7.5 \mathrm{~Hz}, 1 \mathrm{H}), 7.17(\mathrm{~m}, 3 \mathrm{H}) ;{ }^{13} \mathrm{C}$ NMR (50 MHz, $\mathrm{CDCl}_{3}$ ); 22.1 (minor), 22.4 (major), 25.5 (major), 27.1 (major), 27.6 (minor), 27.9 (minor), 32.6 (major), 49.7 (minor), 50.5 (major), 53.3 (minor), 54.8 (major), 74.0 (minor), 74.1 (major), 100.0 (minor), 100.1 (major), 107.2 (major), 107.5 (minor), 112.7 (major), 115.8 (major), 116.8 (minor), 117.1 (major), 117.2 (major), 117.7 (minor), 117.9 (minor), 120.7 (major), 120.9 (major), 121.0 (minor), 121.4 (minor), 125.1 (major), 125.2 (minor), 125.7 (major), 126.0 (minor), 128.8 (minor), 128.9 (major), 131.5 (minor), 131.6 (major), 137.2 (minor), 137.3 (major), 150.4 (minor), 150.5 (major), 168.3 (major), 168.8 (minor); IR (neat) 2938, 2359, $1643 \mathrm{~cm}^{-1}$; HRMS $\left(\mathrm{M}+\mathrm{H}^{+}\right)$calcd for $\mathrm{C}_{23} \mathrm{H}_{25} \mathrm{~N}_{2} \mathrm{O}_{3} 377.1859$, found 377.1850 .
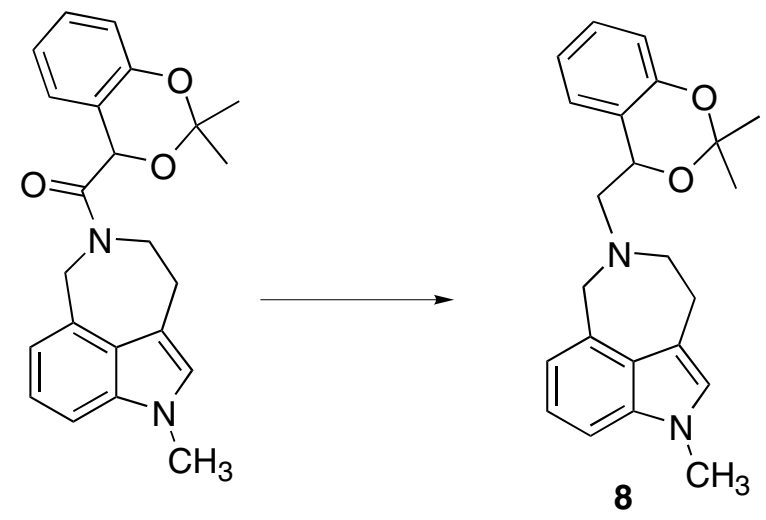

Amine 8. To a solution of lithium aluminum hydride $(66.3 \mathrm{mg}, 1.74 \mathrm{mmol})$ in THF (2.9 $\mathrm{mL}$ ) was added dropwise the amide derived from the previous experimental procedure 
(124 mg, $0.329 \mathrm{mmol})$ in THF $(3.3 \mathrm{~mL})$. The solution was heated at $70{ }^{\circ} \mathrm{C}$ for $3 \mathrm{~h}$. The mixture was quenched with water $(66 \mu \mathrm{L}), 10 \% \mathrm{NaOH}(99 \mu \mathrm{L})$, and water $(198 \mu \mathrm{L})$. The slurry was filtered and concentrated to afford a colorless oil $(119 \mathrm{mg}, 92 \%) ;{ }^{1} \mathrm{H}$ NMR $\left(360 \mathrm{MHz}, \mathrm{CDCl}_{3}\right) \delta 1.51(\mathrm{~s}, 3 \mathrm{H}), 1.63(\mathrm{~s}, 3 \mathrm{H}), 3.06(\mathrm{dd}, J=7.5 \mathrm{~Hz}, 13.8 \mathrm{~Hz}, 1 \mathrm{H}), 3.15$ $(\mathrm{m}, 3 \mathrm{H}), 3.47(\mathrm{t}, J=6.1 \mathrm{~Hz}, 2 \mathrm{H}), 3.76(\mathrm{~s}, 3 \mathrm{H}), 4.44(\mathrm{~d}, J=16.8 \mathrm{~Hz}, 1 \mathrm{H}), 4.49(\mathrm{~d}, J=$ $16.8 \mathrm{~Hz}, 1 \mathrm{H}), 5.13(\mathrm{dd}, J=3.6,7.5 \mathrm{~Hz}, 1 \mathrm{H}), 6.79(\mathrm{dt}, J=1.2,7.5 \mathrm{~Hz}, 1 \mathrm{H}), 6.87(\mathrm{~m}, 3$ $\mathrm{H}), 7.14(\mathrm{~m}, 4 \mathrm{H}) ;{ }^{13} \mathrm{C}$ NMR $\left(50 \mathrm{MHz}, \mathrm{CDCl}_{3}\right) \delta 21.8,25.8,28.4,32.6,55.1,56.9,62.1$, 68.7, 99.4, 107.1, 113.9, 116.8, 117.0, 120.3, 121.3, 122.6, 124.8, 125.7, 126.5, 127.9, 134.9, 137.1, 151.3; IR (neat) 2919, 1609, 1583, 1487, $1458 \mathrm{~cm}^{-1}$; HRMS (M + $\mathrm{H}^{+}$) calcd for $\mathrm{C}_{23} \mathrm{H}_{27} \mathrm{~N}_{2} \mathrm{O}_{2}$ 363.2067, found 363.2057.

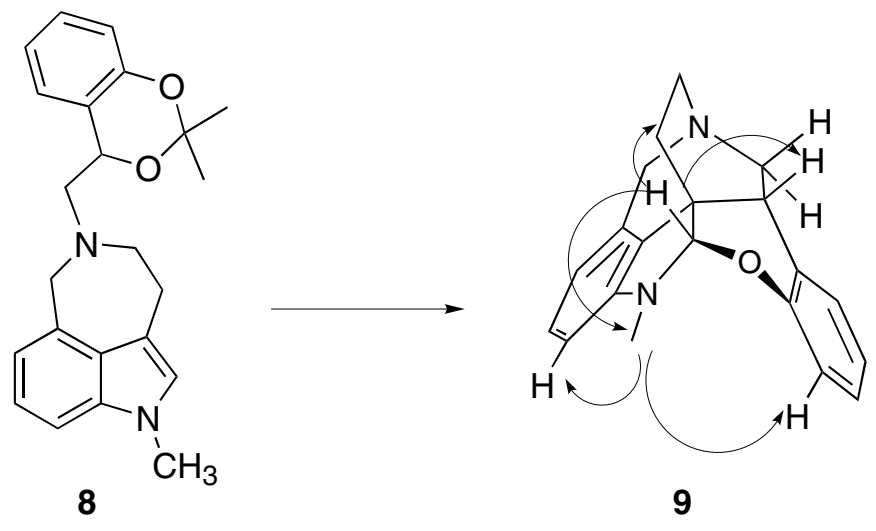

Cycloadduct 9. A solution of the amine 8 (161 $\mathrm{mg}, 0.444 \mathrm{mmol})$ in decahydronaphthalene $(44.4 \mathrm{~mL})$ was heated at $195^{\circ} \mathrm{C}$ for $27 \mathrm{~h}$. Removal of the solvent and purification by silica-gel chromatography $(2: 3$ acetone : hexanes) afforded two diastereomers $(84.5 \mathrm{mg}, 63 \%)$. Major diastereomer $(79 \mathrm{mg}, 59 \%): \mathrm{mp} 153-154{ }^{\circ} \mathrm{C} ;{ }^{1} \mathrm{H}$ NMR (300 MHz, $\left.\mathrm{CDCl}_{3}\right) \delta 2.26(\mathrm{~m}, 2 \mathrm{H}), 2.90(\mathrm{~s}, 3 \mathrm{H}), 3.20(\mathrm{~m}, 2 \mathrm{H}), 3.43(\mathrm{~m}, 2 \mathrm{H})$, $3.78(\mathrm{dd}, J=8.7,13.7 \mathrm{~Hz}, 1 \mathrm{H}), 4.15(\mathrm{~d}, J=17.6 \mathrm{~Hz}, 1 \mathrm{H}), 4.34(\mathrm{~d}, J=17.6 \mathrm{~Hz}, 1 \mathrm{H})$, $5.40(\mathrm{~s}, 1 \mathrm{H}), 5.95(\mathrm{~d}, J=7.6 \mathrm{~Hz}, 1 \mathrm{H}), 6.25(\mathrm{~d}, J=7.6 \mathrm{~Hz}, 1 \mathrm{H}), 6.88(\mathrm{t}, J=7.6 \mathrm{~Hz}, 1 \mathrm{H})$, $6.94(\mathrm{~m}, 3 \mathrm{H}), 7.09(\mathrm{~m}, 1 \mathrm{H}) ;{ }^{1} \mathrm{H}$ NMR $\left(400 \mathrm{MHz}, \mathrm{d}^{6}-\right.$ benzene) $\delta 1.68$ (ddd, $J=3.3,9.3$, $12.3 \mathrm{~Hz}, 1 \mathrm{H}), 1.85(\mathrm{ddd}, J=7.1,9.3,12.3 \mathrm{~Hz}, 1 \mathrm{H}), 2.62(\mathrm{~s}, 3 \mathrm{H}) 2.86(\mathrm{~m}, 1 \mathrm{H}), 2.89$ (t, $J=8.6 \mathrm{~Hz}, 1 \mathrm{H}), 3.06(\mathrm{ddd}, J=7.1,9.3,14.8 \mathrm{~Hz}, 1 \mathrm{H}), 3.34(\mathrm{ddd}, J=2.2,8.6,13.7 \mathrm{~Hz}$, $1 \mathrm{H}), 3.52(\mathrm{dd}, J=8.6,13.7,1 \mathrm{H}), 4.01(\mathrm{~d}, J=17.7 \mathrm{~Hz}, 1 \mathrm{H}), 4.29(\mathrm{~d}, J=17.7 \mathrm{~Hz}, 1$ H), $5.17(\mathrm{~s}, 1 \mathrm{H}), 5.79(\mathrm{~d}, J=7.7 \mathrm{~Hz}, 1 \mathrm{H}), 6.23(\mathrm{~d}, J=7.7 \mathrm{~Hz}, 1 \mathrm{H}), 6.71(\mathrm{t}, J=7.5 \mathrm{~Hz}, 1$ $\mathrm{H}), 6.75(\mathrm{~d}, J=7.5 \mathrm{~Hz}, 1 \mathrm{H}), 6.82(\mathrm{t}, J=7.7 \mathrm{~Hz}, 1 \mathrm{H}), 6.94(\mathrm{t}, J=7.2 \mathrm{~Hz}, 1 \mathrm{H}), 7.03(\mathrm{~d}, J$ $=7.2 \mathrm{~Hz}, 1 \mathrm{H}) ;{ }^{13} \mathrm{C} \mathrm{NMR}\left(50 \mathrm{MHz}, \mathrm{CDCl}_{3}\right) \delta 30.0,38.6,39.0,46.9,48.6,49.3,62.0$, $101.0,101.3,114.0,118.3,122.6,123.9,127.0,128.3,129.6,130.9,136.7,149.6,153.8$; IR (neat) 3228, 1681, 1547, 1502, 1467, 1214, 1190, $1096 \mathrm{~cm}^{-1}$; HRMS (M + $\left.\mathrm{H}^{+}\right)$calcd for $\mathrm{C}_{20} \mathrm{H}_{21} \mathrm{~N}_{2} \mathrm{O} 305.1648$, found 305.1633; Minor diastereomer (5.5 mg, $4 \%$ ); ${ }^{1} \mathrm{H} \mathrm{NMR}$ $\left(400 \mathrm{MHz}, \mathrm{CDCl}_{3}\right) \delta 1.67(\mathrm{~m}, 2 \mathrm{H}), 3.01(\mathrm{~m}, 1 \mathrm{H}), 3.14(\mathrm{~s}, 3 \mathrm{H}), 3.28(\mathrm{~m}, 1 \mathrm{H}), 3.33(\mathrm{t}, J$ $=9.3 \mathrm{~Hz}, 1 \mathrm{H}), 3.51(\mathrm{dd}, J=9.3,13.6 \mathrm{~Hz}, 1 \mathrm{H}), 3.64(\mathrm{br} \mathrm{dd}, J=6.7,13.6 \mathrm{~Hz}, 1 \mathrm{H}), 4.25$ $(\mathrm{d}, J=17.6,1 \mathrm{H}), 4.35(\mathrm{~d}, J=17.6 \mathrm{~Hz}, 1 \mathrm{H}), 4.74(\mathrm{~s}, 1 \mathrm{H}), 6.36(\mathrm{~d}, J=7.7 \mathrm{~Hz}, 1 \mathrm{H}), 6.49$ $(\mathrm{d}, J=7.7 \mathrm{~Hz}, 1 \mathrm{H}), 7.00(\mathrm{dd}, J=1.1,7.6 \mathrm{~Hz}, 1 \mathrm{H}), 7.07$ (dt, $J=1.1,7.6 \mathrm{~Hz}, 1 \mathrm{H}), 7.11$ 
$(\mathrm{t}, J=7.7 \mathrm{~Hz}, 1 \mathrm{H}), 7.16(\mathrm{~d}, J=7.5 \mathrm{~Hz}, 1 \mathrm{H}), 7.23(\mathrm{t}, J=7.5 \mathrm{~Hz}, 1 \mathrm{H}) ;{ }^{13} \mathrm{C}$ NMR $(50$ $\left.\mathrm{MHz}, \mathrm{CDCl}_{3}\right) \delta 29.4,30.7,38.5,46.4,48.6,49.9,59.7,100.8,103.0,115.5,118.0,122.7$, 124.8, 127.6, 128.5, 130.9, 132.9, 153.8; IR (neat) 3228, 1681, 1547, 1502, $1467 \mathrm{~cm}^{-1}$; HRMS $\left(\mathrm{M}+\mathrm{H}^{+}\right)$calcd for $\mathrm{C}_{20} \mathrm{H}_{21} \mathrm{~N}_{2} \mathrm{O} 305.1648$, found 305.1650 .
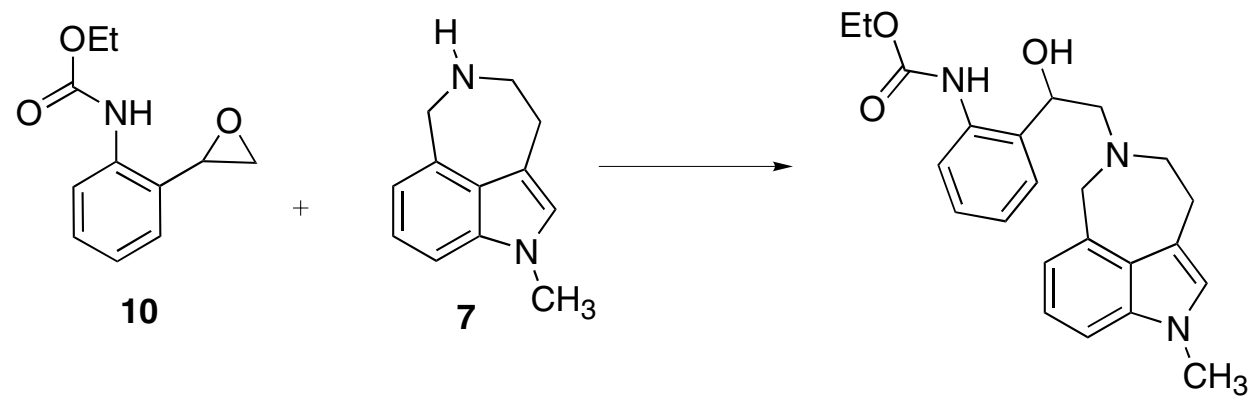

Ring opening of epoxide 10 with benzazepine 7. To a solution of the amine 7 (20.0 $\mathrm{mg}, 0.107 \mathrm{mmol})$ in isopropanol $(110 \mu \mathrm{L})$ was added the epoxide ${ }^{7} \mathbf{1 0}(22.2 \mathrm{mg}, 0.107$ mmol). The solution was stirred at $25^{\circ} \mathrm{C}$ for $12 \mathrm{~h}$ and concentrated to afford a colorless oil as a $9: 1$ mixture of regioisomers, which was used in the next step $(41 \mathrm{mg}, 99 \%) ;{ }^{1} \mathrm{H}$ NMR (360 MHz, $\left.\mathrm{CDCl}_{3}\right) \delta 1.41(\mathrm{t}, J=7.1 \mathrm{~Hz}, 3 \mathrm{H}), 2.93(\mathrm{dd}, J=10.9,12.9 \mathrm{~Hz}, 1 \mathrm{H})$, 3.08 (dd, $J=3.7,12.9 \mathrm{~Hz}, 1 \mathrm{H}), 3.21(\mathrm{q}, J=4.9 \mathrm{~Hz}, 2 \mathrm{H}), 3.46$ (t, $J=4.9,2 \mathrm{H}), 3.84$ (s, 3 H), $4.30(\mathrm{q}, J=7.1 \mathrm{~Hz}, 2 \mathrm{H}), 4.43(\mathrm{~d}, J=16.6 \mathrm{~Hz}, 1 \mathrm{H}), 4.50(\mathrm{~d}, J=16.6 \mathrm{~Hz}, 1 \mathrm{H}), 4.90$ (dd, $J=3.7,10.8,1 \mathrm{H}), 6.96(\mathrm{~m}, 2 \mathrm{H}), 7.01(\mathrm{t}, J=7.4 \mathrm{~Hz}, 1 \mathrm{H}), 7.09$ (dd, $J=1.5,7.7 \mathrm{~Hz}$, $1 \mathrm{H}), 7.27(\mathrm{~m}, 1 \mathrm{H}), 7.30(\mathrm{~m}, 1 \mathrm{H}), 7.34(\mathrm{~m}, 1 \mathrm{H}), 8.11(\mathrm{~d}, J=7.9 \mathrm{~Hz}, 1 \mathrm{H}), 9.04(\mathrm{br} \mathrm{s}, 1$ $\mathrm{H}) ;{ }^{13} \mathrm{C}$ NMR $\left(50 \mathrm{MHz}, \mathrm{CDCl}_{3}\right) \delta 14.6,26.2,32.6,55.2,57.3,60.8,61.5,70.5,107.5$, 113.1, 116.9, 120.6, 121.3, 122.6, 126.0, 127.6, 127.9, 128.4, 133.2, 137.1, 137.6, 153.8; IR (neat) $3318,2911,1727,1591 \mathrm{~cm}^{-1}$; HRMS $\left(\mathrm{M}+\mathrm{H}^{+}\right)$calcd for $\mathrm{C}_{23} \mathrm{H}_{28} \mathrm{~N}_{3} \mathrm{O}_{3} 394.2125$, found 394.2111 .
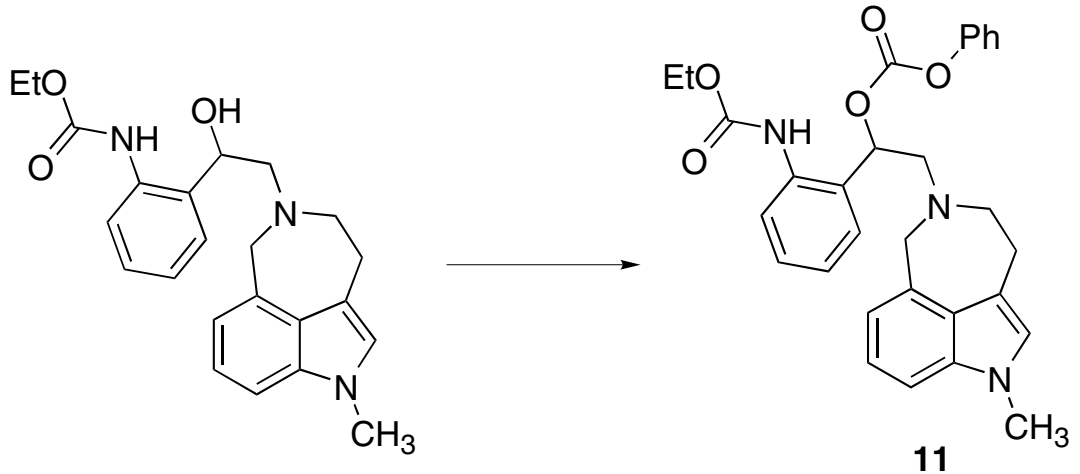

Carbonate 11. To a solution of the alcohol $(25.8 \mathrm{mg}, 0.066 \mathrm{mmol})$ in $\mathrm{CH}_{2} \mathrm{Cl}_{2}(220 \mu \mathrm{L})$ at $0{ }^{\circ} \mathrm{C}$ was added pyridine $(16.0 \mu \mathrm{L}, 0.197 \mathrm{mmol})$ and phenyl chloroformate $(12.3 \mu \mathrm{L}$, $0.098 \mathrm{mmol}$ ). The solution was warmed to room temperature over $1.5 \mathrm{~h}$. The mixture was quenched with water and extracted with $\mathrm{CH}_{2} \mathrm{Cl}_{2}$. The combined organic extracts 
were dried $\left(\mathrm{Na}_{2} \mathrm{SO}_{4}\right)$, filtered, and concentrated. Purification by silica-gel chromatography (1:3 ethyl acetate : hexanes) provided a yellow oil $(22 \mathrm{mg}, 65 \%) ;{ }^{1} \mathrm{H}$ NMR $\left(360 \mathrm{MHz}, \mathrm{CDCl}_{3}\right) \delta 1.22(\mathrm{t}, J=7.1 \mathrm{~Hz}, 3 \mathrm{H}), 3.08(\mathrm{dd}, J=7.6,13.8 \mathrm{~Hz}, 1 \mathrm{H})$, $3.13(\mathrm{t}, J=5.4 \mathrm{~Hz}, 2 \mathrm{H}), 3.34(\mathrm{~m}, 3 \mathrm{H}), 3.75(\mathrm{~s}, 3 \mathrm{H}), 4.14(\mathrm{dq}, J=1.6,7.1 \mathrm{~Hz}, 2 \mathrm{H}), 4.31$ $(\mathrm{d}, J=16.6 \mathrm{~Hz}, 1 \mathrm{H}), 4.39$ (d, $J=16.6 \mathrm{~Hz}, 1 \mathrm{H}), 6.03(\mathrm{dd}, J=4.5,7.6 \mathrm{~Hz}, 1 \mathrm{H}), 6.83(\mathrm{~d}, J$ $=6.7 \mathrm{~Hz}, 1 \mathrm{H}), 6.86(\mathrm{~m}, 1 \mathrm{H}), 7.13(\mathrm{~m}, 4 \mathrm{H}), 7.18(\mathrm{~d}, J=7.3 \mathrm{~Hz}, 1 \mathrm{H}), 7.24(\mathrm{brt}, J=7.5$ $\mathrm{Hz}, 1 \mathrm{H}), 7.36(\mathrm{~m}, 3 \mathrm{H}), 7.42(\mathrm{dd}, J=1.5,7.8 \mathrm{~Hz}, 1 \mathrm{H}), 7.84(\mathrm{~d}, J=7.6 \mathrm{~Hz}, 1 \mathrm{H}), 9.43$ (br $\mathrm{s}, 1 \mathrm{H}) ;{ }^{13} \mathrm{C} \mathrm{NMR}\left(50 \mathrm{MHz}, \mathrm{CDCl}_{3}\right) \delta 14.4,25.1,32.6,55.0,56.9,60.8,61.8,74.9,107.5$, 113.2, 117.4, 120.9, 121.3, 122.8, 124.1, 125.9, 126.0, 126.2, 126.3, 128.8, 129.4, 130.2, 133.1, 136.0, 137.0, 151.0, 153.0, 154.4; IR (neat) 2910, 1759, 1727, $1251 \mathrm{~cm}^{-1}$; HRMS $\left(\mathrm{M}+\mathrm{H}^{+}\right)$calcd for $\mathrm{C}_{30} \mathrm{H}_{32} \mathrm{~N}_{3} \mathrm{O}_{5}$ 514.2336, found 514.2309.
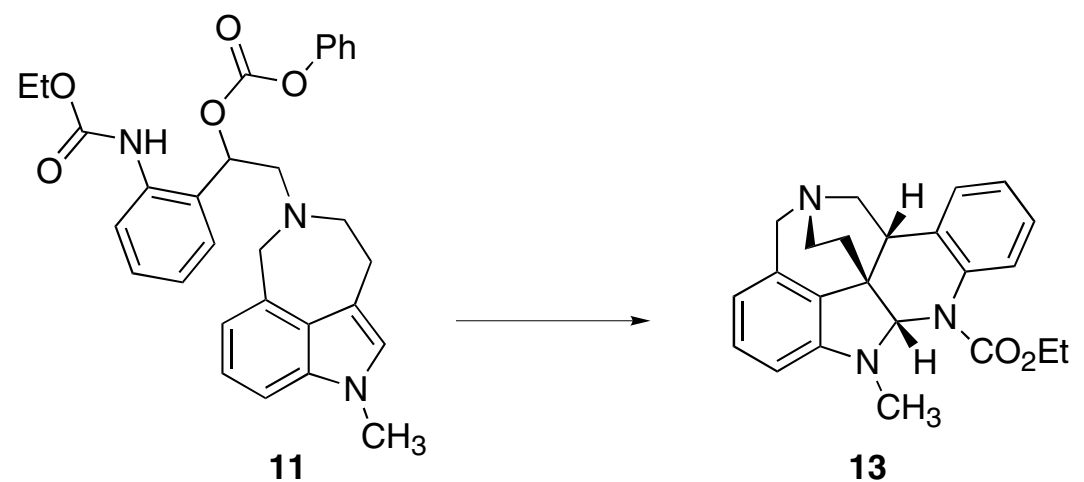

Cycloadduct 13. A solution of the carbonate $11(10.0 \mathrm{mg}, 0.019 \mathrm{mmol})$ in 1,2dichlorobenzene $(1.9 \mathrm{~mL})$ was heated at $160{ }^{\circ} \mathrm{C}$ for $6 \mathrm{~h}$. Removal of the solvent and purification by silica-gel chromatography $\left(1: 19 \mathrm{MeOH}: \mathrm{CH}_{2} \mathrm{Cl}_{2}\right)$ provided a yellow oil (5 mg, $70 \%) ;{ }^{1} \mathrm{H}$ NMR $\left(360 \mathrm{MHz}, \mathrm{CDCl}_{3}\right) \delta 1.31(\mathrm{~m}, 3 \mathrm{H}), 2.19(\mathrm{t}, J=10.7 \mathrm{~Hz}, 1 \mathrm{H})$, $2.42(\mathrm{~m}, 1 \mathrm{H}), 2.74(\mathrm{~s}, 3 \mathrm{H}), 3.02(\mathrm{t}, J=8.5 \mathrm{~Hz}, 1 \mathrm{H}), 3.17(\mathrm{~m}, 1 \mathrm{H}), 3.41(\mathrm{~m}, 2 \mathrm{H}), 3.71$ $(\mathrm{dd}, J=5.6,14.0 \mathrm{~Hz}, 1 \mathrm{H}), 4.14(\mathrm{~d}, J=17.8 \mathrm{~Hz}, 1 \mathrm{H}), 4.31(\mathrm{~d}, J=17.8 \mathrm{~Hz}, 1 \mathrm{H}), 4.34$ $(\mathrm{m}, 2 \mathrm{H}), 5.90(\mathrm{~d}, J=7.7 \mathrm{~Hz}, 1 \mathrm{H}), 5.90(\mathrm{br} \mathrm{s}, 1 \mathrm{H}), 6.23(\mathrm{~d}, J=7.7 \mathrm{~Hz}, 1 \mathrm{H}), 6.86(\mathrm{t}, J=$ $7.7 \mathrm{~Hz}, 1 \mathrm{H}), 7.02(\mathrm{~d}, J=7.3 \mathrm{~Hz}, 1 \mathrm{H}), 7.05(\mathrm{t}, J=7.3 \mathrm{~Hz}, 1 \mathrm{H}), 7.16(\mathrm{t}, J=7.3 \mathrm{~Hz}, 1 \mathrm{H})$, 7.26 (v br s, $1 \mathrm{H}) ;{ }^{1} \mathrm{H}$ NMR $\left(360 \mathrm{MHz}, \mathrm{CD}_{3} \mathrm{CN}\right) \delta 1.25(\mathrm{br} \mathrm{m}, 3 \mathrm{H}), 2.09(\mathrm{~m}, 1 \mathrm{H}), 2.28$ $(\mathrm{m}, 1 \mathrm{H}), 2.71(\mathrm{~s}, 3 \mathrm{H}), 3.01(\mathrm{t}, J=8.7 \mathrm{~Hz}, 1 \mathrm{H}), 3.08$ (ddt, $J=2.5,9.7,14.3 \mathrm{~Hz}, 1 \mathrm{H})$, $3.25(\mathrm{~m}, 1 \mathrm{H}), 3.37$ (ddd, $J=2.5,8.7,13.7 \mathrm{~Hz}, 1 \mathrm{H}), 3.61(\mathrm{dd}, J=8.7,13.7 \mathrm{~Hz}, 1 \mathrm{H})$, $4.05(\mathrm{~d}, J=17.9 \mathrm{~Hz}, 1 \mathrm{H}), 4.21(\mathrm{~d}, J=17.9 \mathrm{~Hz}, 1 \mathrm{H}), 4.26(\mathrm{br} \mathrm{m}, 2 \mathrm{H}), 5.81(\mathrm{br} \mathrm{s}, 1 \mathrm{H})$, $5.90(\mathrm{~d}, J=7.7 \mathrm{~Hz}, 1 \mathrm{H}), 6.20(\mathrm{~d}, J=7.7 \mathrm{~Hz}, 1 \mathrm{H}), 6.81(\mathrm{t}, J=7.7 \mathrm{~Hz}, 1 \mathrm{H}), 7.06(\mathrm{~m}, 2$ $\mathrm{H}), 7.17(\mathrm{~m}, 1 \mathrm{H}), 7.33$ (br s, $1 \mathrm{H}) ;{ }^{13} \mathrm{C} \mathrm{NMR}\left(90 \mathrm{MHz}, \mathrm{CDCl}_{3}\right) \delta 14.9,30.1,30.9,40.4$, $41.3,47.5,49.5,51.5,62.3,62.4,83.4,102.1,114.3,124.1,125.9,126.1,126.7,128.9$, 129.8, 134.9, 137.4, 137.7, 150.4; IR (neat) 2939, 1642, 1487, $1458 \mathrm{~cm}^{-1}$; HRMS (M + $\mathrm{H}^{+}$) calcd for $\mathrm{C}_{23} \mathrm{H}_{26} \mathrm{~N}_{3} \mathrm{O}_{2} 376.2019$, found 376.2022. 


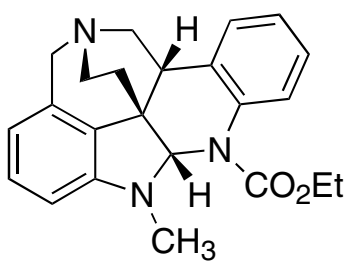

13

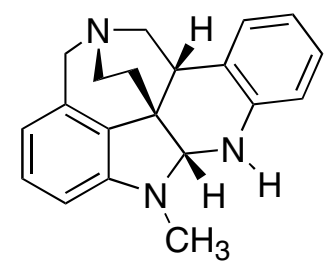

14

Amine 14. To a solution of the carbamate $13(14.6 \mathrm{mg}, 0.039 \mathrm{mmol})$ in ethylene glycol $(389 \mu \mathrm{L})$ was added $\mathrm{KOH}(56.0 \mathrm{mg}, 1.01 \mathrm{mmol})$ and hydrazine $(9.1 \mu \mathrm{L}, 0.19 \mathrm{mmol})$. The solution was heated at $150{ }^{\circ} \mathrm{C}$ for $4 \mathrm{~h}$. The mixture was quenched with $\mathrm{H}_{2} \mathrm{O}$ and extracted with $\mathrm{CHCl}_{3}$. The combined organic extracts were dried $\left(\mathrm{Na}_{2} \mathrm{SO}_{4}\right)$, filtered, and concentrated. Purification by silica-gel chromatography (1: $15 \mathrm{MeOH}: \mathrm{CH}_{2} \mathrm{Cl}_{2}$ ) provided a yellow oil $(8 \mathrm{mg}, 67 \%) ;{ }^{1} \mathrm{H} \mathrm{NMR}\left(400 \mathrm{MHz}, \mathrm{CDCl}_{3}\right) \delta 2.19(\mathrm{ddd}, J=2.1$, 9.1, $11.5 \mathrm{~Hz}, 1 \mathrm{H}), 2.35$ (ddd, $J=9.1,12.4,17.7 \mathrm{~Hz}, 1 \mathrm{H}), 2.72$ (s, $3 \mathrm{H}), 2.98$ (br t, $J=$ $9.1 \mathrm{~Hz}, 1 \mathrm{H}), 3.17(\mathrm{~m}, 1 \mathrm{H}), 3.35(\mathrm{t}, J=8.2 \mathrm{~Hz}, 1 \mathrm{H}), 3.37(\mathrm{~m}, 1 \mathrm{H}), 3.75(\mathrm{dd}, J=8.2$, $13.3 \mathrm{~Hz}, 1 \mathrm{H}), 4.15(\mathrm{~d}, J=17.7 \mathrm{~Hz}, 1 \mathrm{H}), 4.35$ (d, $J=17.7 \mathrm{~Hz}, 1 \mathrm{H}), 4.53(\mathrm{~d}, J=2.1 \mathrm{~Hz}$, $1 \mathrm{H}), 4.63$ (br s, $1 \mathrm{H}), 5.95(\mathrm{~d}, J=7.7 \mathrm{~Hz}, 1 \mathrm{H}), 6.26(\mathrm{~d}, J=7.7 \mathrm{~Hz}, 1 \mathrm{H}), 6.71(\mathrm{~d}, J=7.6$ $\mathrm{Hz}, 1 \mathrm{H}), 6.79(\mathrm{t}, J=7.6 \mathrm{~Hz}, 1 \mathrm{H}), 6.90(\mathrm{br} \mathrm{t}, J=7.7 \mathrm{~Hz}, 2 \mathrm{H}), 7.04(\mathrm{t}, J=7.6 \mathrm{~Hz}, 1 \mathrm{H})$;

${ }^{13} \mathrm{C}$ NMR (50 MHz, $\left.\mathrm{CDCl}_{3}\right) \delta 30.8,40.5,40.9,47.2,49.3,50.0,61.9,84.4,101.7,113.9$, $116.2,120.5,123.8,126.6,128.3,130.0,130.1,137.1,143.9,150.7$; IR (neat) 2851, 1597, $1492 \mathrm{~cm}^{-1}$; HRMS $\left(\mathrm{M}+\mathrm{H}^{+}\right)$calcd for $\mathrm{C}_{20} \mathrm{H}_{22} \mathrm{~N}_{3} 304.1808$, found 304.1829.

\section{References}

1. We followed the procedure for chiral glyoxylates, see: Bigi, F.; Bocelli, G.; Maggi, R.; Sartori, G. J. Org. Chem. 1999, 64, 5004.

2. Clark, R. D.; Weinhardt, K. K.; Berger, J.; Fisher, L. E.; Brown, C. M.; MacKinnon, A. C.; Kilpatrick, A. T.; Spedding, M. J. Med. Chem. 1990, 33, 633.

3. Kitano, M.; Kojima, A.; Nakano, K.; Miyagashi, A.; Noguchi, T.; Ohashi, N. Chem. Pharm. Bull. 1999, 47, 1538.

4. Flaugh, M. E.; Crowell, T. A.; Clemens, J. A.; Sawyer, B. D. J. Med. Chem. 1979, 22, 63.

5. Strekowski, L.; Van Aken, K.; Gulevich, Y. J. Heterocyclic Chem. 2000, 37, 1495.

6. Evans, D. D.; Bowman, R. E.; Nagy, H.; Guyett, J.; Weale, J.; Weyell, D. J.; White, A. C. J. Chem. Soc., Perkin Trans. 1, 1972, 1926.

7. Ruano, J. L. G.; Pedrega, C.; Rodriguez, J. H. Tetrahedron 1989, 45, 203. 